\title{
Determine Characters by Mathematical Model for Segmentation Arabic Words by Voronoi Diagrams
}

\author{
Jabril Ramdan, Khairuddin Omar and Mohammad Faidzul \\ School of Computer Science, Faculty of Information Science and Technology, Centre of Artificial Intelligence \\ Universiti Kebangsaan Malaysia, Bangi - 43200, Selangor, Malaysia
}

\begin{abstract}
Objectives: The objectives are to use a mathematical model to define a region-based segmentation method. This study determines whether the Connected Component (CC) is one or more than one character. Method: Whereas the other methods they tend to ignore the solid foundation of describing characters and connection points. This proposed method adopts on many stages for adaptive the mathematic in segmentation characters process are: i) peak detection from vertical histogram for (CC), and ii) enhancement of the model using a mathematical model to improve the segmentation method based on the Voronoi Diagram (VD) Through a number of peaks. Findings: Whereas characters, such as w and ص, are confusing to segmentation methods; these errors include separating connection strokes from both sides to produce a separated one. Other errors must be handled at a later stage, such as segmenting the character $\tau-$ at an acute angle. Whereas the mathematical model is depending on peaks, numbers, direction, and length of CC. This model is tested on segmentation using five Arabic datasets as: AHDB, IFN-ENIT, AHDB-FTR, APTI, Zeki and Al Hamad DB datasets. The Preliminary results show that the application of the EDMS feature with multi perceptron-NN classifier it's preferable. Its accuracy when compared with Zeki method is $\mathbf{9 6 . 8 1 \%}$ for the ACTOR printed dataset and the rate of this method is $\mathbf{8 5 . 8 1 \%}$ for Zeki dataset and also compared with Al Hamad method is $95.09 \%$, and $89.10 \%$ for ACDARhandwrittendataset. Whereas the others datasets accuracies are $\mathbf{9 5 . 0 9 \%}$ for IFN-ENIT, $\mathbf{9 8 . 2 7 \%}$ for APTI, $\mathbf{9 1 . 6 3 \%}$ for AHDB, and $\mathbf{9 0 . 6 9 \%}$ for AHDB-FTR on same feature (EDMS) and classifier (MLP_NN). Novelty: Adapt Mathematics with segmentation process to determine whether the $\mathrm{CC}$ is one or more than one character. Using a mathematical model based on the VD to avoid over segmentation .
\end{abstract}

Keywords: Mathematical Model, Arabic words, More than one character, Segmentation, Voronoi Diagrams

\section{Introduction}

Due to the uniqueness of the characters of the Arabic/Jawi language, there is a high incidence of error when pixelbased methods of character recognition are employed. When applying these methods, it is common to ignore the foundation of a character's description in these languages because Arabic/Jawi is typically segmented into multiple stages in recognition systems. The language is difficult to analyze using pixel-based methods; indeed, segmentation has been proven to be particularly challenging, and it is likely that characters will be over-segmented due to the many bulges in certain characters, including seen (w), lam-alif (ال), and sad (ص), producing errors when they are separated. Such errors are generated in the Voronoibased recognition method, even though this method makes great effort to minimize over-segmentation using several rules, such as in the case of the character $\omega^{1,4}$. The challenges of the arc system are presented in this critical segmentation phase.

The reason that this challenge exists is primarily due to the complexity of the image shapes of the characters that constitute this language. The VD algorithm involves two primary parameters, the speed and quality of a VD construction. Because this method produces the best structures during segmentation, a construction algorithm is viewed as the most feasible link between the static and dynamic styles of algorithm development; thus, this

\footnotetext{
*Author for correspondence
} 
method, known as the divide-and-conquer algorithm, has been chosen ${ }^{2}$.

According ${ }^{3}$ states that the primary purpose of oversegmentation is to divide characters into single entities such that no merged characters remain after the process. Discarding invalid points of segmentation and retaining valid points of segmentation are considered to be two of the primary problems following over-segmentation.

This paper demonstrates novel techniques for detecting whether connected components represent one character or more than one character to avoid over-segmentation and to reduce the computation time required for the segmentation of letters in the Arabic language. The proposed method is supported by mathematical models and examples that address the scarcity of such information in the literature, despite the use of existing handwritten databases. [Figure 1] shows the types uses of datasets in this article.

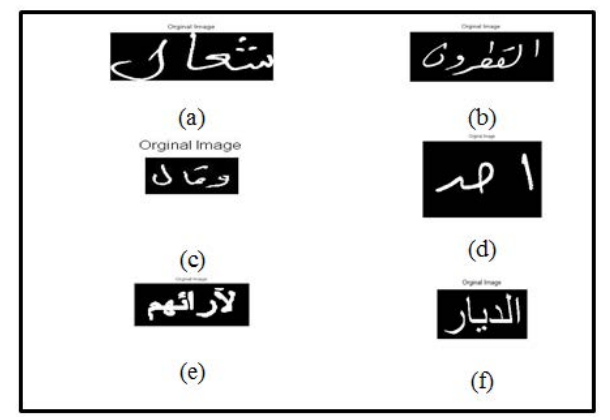

Figure 1. Examples of datasets: (a) IFN/ENIT, (b) AHDB/ FTR, (c) Al Hamad, (d) AHDB, (e) APTI, and (f) Zeki.

\section{Character Segmentation Problem and Weaknesses}

Through the present methods applied for oversegmentation., that of $^{3}$, is clearly optimal. However, considering the different failure of the available techniques, which contain thinning, a new technique for boost the heuristic algorithm to decrease "bad" errors and increase total outcome accuracy is eligible. An insightful and more helpful technique for identifying the range of a component as a separate segment of a bigger character may be acquired by utilize a connected component's width-to-height ratio. To ensure a higher rate of accuracy in classifying components, more complex heuristics must be developed based on features that exclude aspect ratios and positioning of these components.
Arabic is currently one of the most widespread languages in the world, and several robust studies on the recognition of its handwritten script have been reported. However, to date, none of these studies has been comprehensive because Arabic is considerably more complex than Latin languages. [Figure 2] illustrates some of complexity of Arabic characters. The problems associated with the segmentation of Arabic scripts into characters and their classifications make Arabic even more difficult to study ${ }^{3}$.

There are several issues associated with segmentation and methods thereof. In some languages, certain characters contain strokes or bulges that easily and effectively can be segmented by many of the available segmentation methods, a property referred to as oversegmentation, ${ }^{1,17}$ :

- Characters that have the primary shape $\tau$-and acute angles within a character tend to be segmented incorrectly.

- Characters with several bulges, such as س and may be segmented as one unit.

- The primary issue associated with the character $\operatorname{e}$ is that the segmentation of the character frequently occurs at its corner below the loop.

- Segmentation is considered to separate the tails of the primary shapes ص ص $س$, and, producing the $u$ shape in addition to the first part(s) of the character.

- A hanging segment is the result of segmenting tails of certain characters, such as $ب, \dot{\omega}$, , and $\mathrm{J}$.

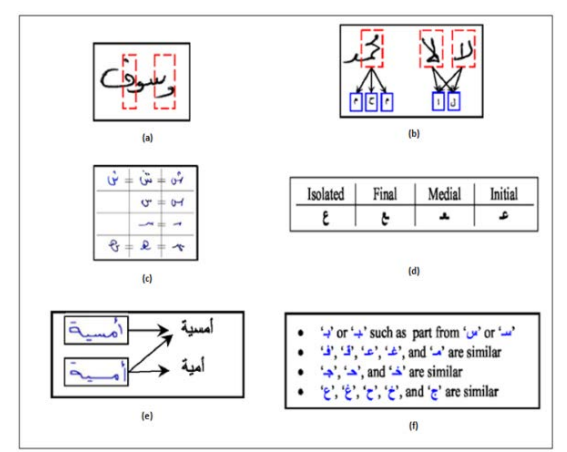

Figure 2. Shapes of Arabic characters in different positions, fonts, overlapping, shared horizontal space: (a) two characters occupying a shared horizontal space, (b) Arabic ligatures, their constituent characters, (c) four characters written in completely different ways, (d) shapes of the character " $\varepsilon$," (e) letter " may be missed, and (f) characters with similar contours. 


\section{Proposed Method}

The purpose of the suggest method is to beat the problems and failure of the prior methods. Therefore, we developed a high-performance method that is generally eligible of qualify all challenges related by over segmentation, and is particularly effective in cases in which the prior failed methods. The suggest method assume a local approach and is combined of two method: an orientation method for define connected components in two directions (left, right) based on the features of an image, and a novel method that can define the proper value the thresholding of every peaks along the $y$-axis and the length connected components along the $\mathrm{x}$-axis.

\subsection{Architecture}

The proposed method follows the steps shown in [Figure 3].

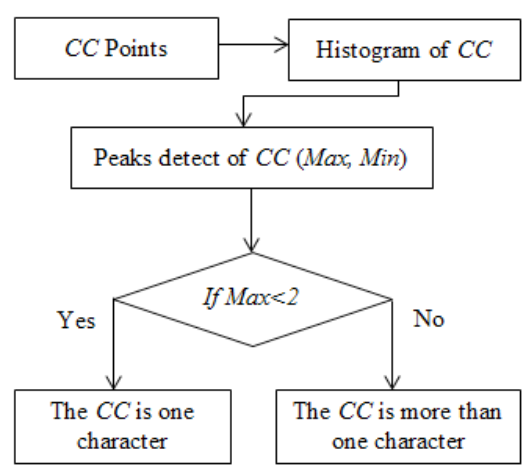

Figure 3. Illustration of determination of CC of one or more than one character.

\subsection{Vertical Projection Profile (Histogram)}

Vertical Projection profile is performed by counting the number of black pixels in every image row using the following formula:

$V p p_{v}(x)=\int_{y 1}^{y z} I(x, y) d y$

Vertical Projection Profile (VPP) is a column vector of the image comprising of total of ON pixels for every column. Even Projection Profile (HPP) is the one dimensional column vector cluster alluding to total of ON pixels in the picture for every row ${ }^{12}$.The mathematical representation of the vertical histogram for connected component (اعس) is as shown in [Figure 4].

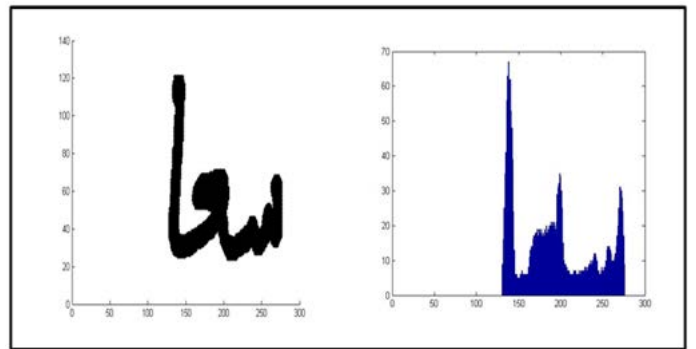

Figure 4. Illustration of vertical histogram for connected component (اعس).

\subsection{Peak Detection}

According to ${ }^{13}$, the peak detected takes two case: the first its values to be evaluated and second its threshold. The first argument is the $y$-values for the maximum of each Colum in the carve, and threshold value its relies on the specific requisition. The peak detected function checks for each value the values to the left and to the right of the considered value. If the behold value overtake the values to the left and right by at minimum the threshold value, then the considered value is count a peak.

Based on this logic, the function should returning three maximum values for the graph created if a proper threshold value is applied. The peak detection function then finding two vectors consisting of all maximum points and all minimum points, as shown in [Figure 5]. If the peak detect is miss or zero in the worst condition, then the connected component is one character, ligature, or dots.

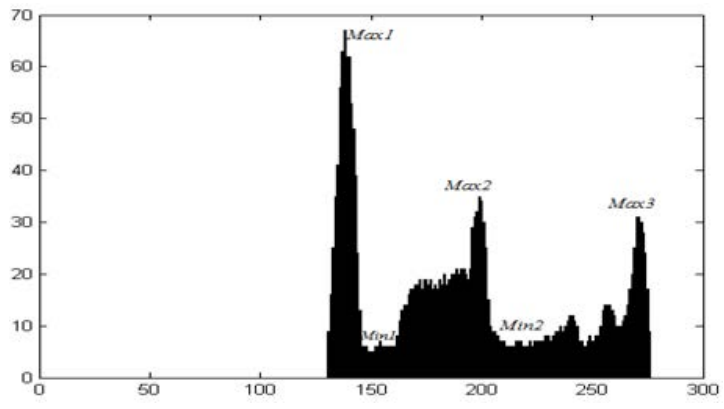

Figure 5. (a) Illustration of the maximum and minimum peaks.

\subsection{Mathematical Model}

The practical world's mathematical applications can be interpreted through mathematical modeling. Alongside, 
Table 1. Specifications of the datasets used in this study

\begin{tabular}{lllllll}
\hline & IFNENIT & APTI & ACDAR & AHDB & Zeki DB & AHDB/FTR \\
\hline $\begin{array}{l}\text { No. of images } \\
\text { No. of writers }\end{array}$ & 596 & 50 & 500 & 3045 & 50 & 497 \\
$\begin{array}{l}\text { Printed /Handwrite } \\
\text { Contents }\end{array}$ & Nandwrite & Nrinted & Handwrite & Handwrite & Printed & Handwrite \\
& Tunisian & Most common & Most common & Most common & Most common Libyan Towns \\
Towns Names & & & & Names \\
Noise & Max.3 words & One word & One word & One word & One word & Max. 3 words \\
& Free & No/need & No/need & Free & Free & No/need \\
Resolution (dpi) & filtering & filtering & & & filtering \\
File format & Mono-chrome & Mono-chrome & Mono-chrome & Mono-chrome & Mono-chrome & Mono-chrome \\
& BMP & PNG & JEPG & TIFF & BMP & BMP \\
\hline
\end{tabular}

mathematical modeling is also used for scrutinizing significant queries about the linked world, elucidating them, idea testing and anticipating with the future conditions in regard to the world practical applications. The fact cannot be neglected that the practical world is linked with sports, wildlife management, chemistry, engineering, computers, physics, ecology, economics, and physiology, etc.

for $i=1,2, \ldots, n$ Where $i$ is number of maximum peaks.

Thus, the following is true:

$f(x)=(i-1) i$

$f(x)=\left\{\begin{array}{ccc}0 & \text { if } & i<2 \\ \geq & 1 & \text { otherwise }\end{array}\right.$

Where 1 is a connected component that is composed of more than one character and 0 is a connected component that comprises only one character.

\subsection{Pseudo Code of Algorithm}

While $\mathrm{c}<$ Connected Components (image)

$\left[\max _{\text {peak }}, \min _{\text {peak }}\right]=$ peakdet (Vertical Histogram, Threshold);

$$
\text { Ifmax }_{\text {peak }}<2 \text { THEN }
$$

Connected Component is One Character;

Else

Connected Component is more than One Character;

Go to next algorithm for segmentation path

EndIf

$\mathrm{c}=\mathrm{c}+1$;

EndWhile

\section{Datasets}

In this research, six databases were applied to estimate the proposed algorithm and segmentation algorithm, inclusive the IFN/ENIT ${ }^{6}, \mathrm{APTI}^{10}, \mathrm{ACDAR}^{8}, \mathrm{AHDB}^{9}$, Zeki primary shapes dataset Al-Jazeera.net ${ }^{1}$, and the AHDB/ FTR $^{7}$. [ Table 1] shows the specifications of the different datasets.

\section{Result and Discussion}

Two types of tests were conducted for this research. The first, a visual test, qualified the images and the outcomes of segmentation. This test was proceeding on choice images that presented different types of segmentation challenges. The second test was an analytical test that provided a statistical measurement based on benchmark datasets and estimation.

\subsection{Visual experiment}

To demonstrate fully the visual performance of this approach, problems such as variance in text size, considerable varying illumination, low image resolution, thin pen stroke lines and minimum disparity among the text and background were combined to the test. Six samples were composed from different sources for this test. [Figures 6(a), (b), (c), and (d)] show various sizes and fonts of the handwritten and printed text samples collected and imported from IFNENIT, AHDB, and APTI; highquality printed samples successively collected from the primary shapes dataset ${ }^{1}$, are shown in [Figures. 6(e) and 
(f)]. To evaluate the performance of the proposed method, we compared its results with those of Zeki's method. The author outlined the factor values of the method based on his own values. It should be noted that the proposed method demonstrated the best results for all chosen challenges, as shown in the first image in [Figure 6(a)]. The most comprehensive results were obtained using the proposed method with the handwritten image, as shown in Figure 6(f), as well as words, as shown in [Figure 6(b)]. Finally, [Figure 6(c)] shows that the different datasets provided better results compared with those shown in [Figure 6(e)].

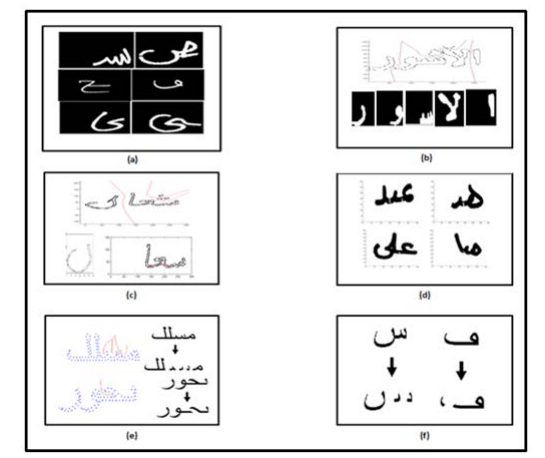

Figure 6. Sample results for (a) segmentation after determining connected components as either one or more than one character from the IFNENIT dataset, (b) a word from the APTI dataset, (c) a word from the IFNENIT dataset with the proposed method's results, and (d) words from the AHDB dataset from the proposed method, and (e) and (f) the primary shape dataset and the Ahmed Zeki algorithm.

\subsection{Analytical Experiment}

Basic features may be recognize similarly as character strokes, character openings, or other character properties, for instance, concavities and convexities, end centers also, crossing points, extreme, union with straight lines et cetera ${ }^{14}$. This test was mostly complete to explain the analytical achievement of the suggested method for various types of segmentation challenges. Therefore, the benchmark datasets used were IFNENIT, AHDB, and APTI. The results obtained after applying the proposed methodology were used to determine whether one or more than one character had been applied to several datasets after the segmentation algorithm. As previously stated, the proposed method appears to produce good results when identifying Arabic characters. [Table 2] presents the findings obtained through the application of three types of feature extraction methods, including edge direction matrixes (EDMS), which is suggested for analysis in terms of the statistical texture method via binary images. Numerous equations were suggested to include characteristics from EDM1 and EDM2 values. By calculating their correlation, the proposed equations extracted 22 features. Homogeneity, pixel regularity, weight, edge direction, and edge regularity, based on the study by ${ }^{15}$, and grey-level co-occurrence matrix (GLCM) texture measurements were used to describe image texture, as proposed by Haralick in the $1970 \mathrm{~s}^{14}$. The invariant moment technique is another local feature extraction method proposed by Hu 1962s. Hu's method features seven invariant moments ${ }^{17}$, and was applied to the three types of classifiers, including a random forest classifier, multiple perceptron neural network, and Rules Ridor. Note that this method used an inverted filter with GLCM and MOMENT features.

Using experiments to evaluate the results obtained from several types of databases and the application of three types of feature extraction methods with three types of classifiers, we found that the best results were obtained with the EDMS feature extraction method with classifier multilayer neural networks, as shown in Table 2. The use of EDMS with MLP-NN was observed to be more effective than the use of GLCM and MOMENT in the other category $(95.09 \%$ in the IFN-ENIT handwritten dataset and $98.27 \%$ in the APTI printed dataset). The GLCM feature extraction method showed lower detection percentages for rule classifiers than did the EDMS and MOMENT methods $(69.43 \%$ in the IFN-ENIT dataset and $71.42 \%$ in the APTI dataset).

For any method some cases its bad or incorrect segmentation; in this method also some cases it performs badly because some reasons affected on result segmentation like different size of fonts for handwritten this happens by reason of fixed threshold of peaks detection, also overlapping characters can affected, and segment some characters when the writing time Although he was supposed to write these characters

As a one body.Comparisons show that the EDMS feature extraction method identifies character components as either one or more than one character using two methods: the Zeki method and histogram. The results demonstrate that the proposed method is effective in avoiding over-segmentation and yields satisfactory results. [Table 3] compares the success rates, locations of segmentation points, over-segmentation, and error rates. 
A simple analysis of variance of the printed and text datasets for the three methods, Zeki, histogram, and the proposed method was performed. The use of this statistic to test the hypothesis formulated in this study revealed that there was no significant difference between the use of any of these three methods when the data generated were compared to those obtained under the alternate hypothesis that states that there is indeed a marked difference in the use of these three methods based on the data generated. [Table 4] and [Table 5] are summarize the results of the single-factor ANOVA.

Based on the p-value shown in [Table 5], it can be concluded that the three methods used differ significantly with respect to their generated values.

\section{Conclusion}

The primary focus of this study was to determine whether an identified component is either a single Arabic character or more than one character to reduce the time required for segmentation and to avoid over-segmentation of characters, as mentioned previously in the theses of ${ }^{1,3,4,17}$. A mathematical model was used to determine whether a connected component is a character that does not require segmentation or more than one character that requires segmentation. The results obtained using this method appears to be promising and reliable for both handwriting and print datasets. The proposed method also possesses none of the weaknesses of previously developed methods described herein. To evaluate the proposed method, we compared it with many other established methods, including those proposed by ${ }^{3,17}$. Both visual and analytical experiments were applied to selected datasets, namely IFN/ENIT, APTI, ACDAR, AHDB, Zeki DB, and AHDB/ FTR. Based on these experiments, the proposed method was observed to yield better performance compared to that of the Zeki and Al Hamad methods. The proposed method solved the problem of identifying connected components and whether they formed one or more than one character. The proposed method is highly adaptable

Table 2. Experimental results from the proposed method

\begin{tabular}{llcccccc}
\hline $\begin{array}{l}\text { Classifier } \\
\text { Types }\end{array}$ & Feature Type & \multicolumn{7}{c}{ DATA SETS } \\
\cline { 3 - 8 } & & IFNENIT & APTI & ACDAR & AHDB & Zeki DB & AHDB_ \\
& & DB & DB & DB & DB & & FTRDB \\
\hline Trees. Ran- & EDMS & 93.962 & 97.413 & 85.643 & 90.909 & 96.015 & 89.767 \\
dom Forest & GLCM & 73.437 & 80.952 & 72.727 & 73.684 & 85.001 & 69.829 \\
& MOMENT & 68.679 & 88.185 & 72.449 & 68.750 & 86.345 & 69.544 \\
Functions. & EDMS & 95.094 & 98.275 & 89.108 & 91.636 & 96.812 & 90.697 \\
Multilayer & GLCM & 71.698 & 72.222 & 62.505 & 60.004 & 72.002 & 59.005 \\
Perceptron & MOMENT & 70.892 & 76.793 & 65.007 & 65.625 & 72.690 & 63.177 \\
Rules. Ridor & EDMS & 90.188 & 96.120 & 77.722 & 89.090 & 91.235 & 85.116 \\
& GLCM & 69.434 & 71.428 & 65.217 & 60.312 & 72.413 & 69.586 \\
& MOMENT & 70.754 & 80.168 & 76.530 & 73.010 & 78.313 & 75.570 \\
\hline
\end{tabular}

Table 3. Result values for three methods

\begin{tabular}{lccc}
\hline & $\begin{array}{c}\text { Proposed } \\
\text { Method }\end{array}$ & $\begin{array}{c}\text { Zeki } \\
\text { Method }\end{array}$ & $\begin{array}{c}\text { Projection } \\
\text { Method }\end{array}$ \\
\hline $\begin{array}{l}\text { Location of Seg- } \\
\text { mentati-on point }\end{array}$ & 94.88 & 80.04 & 57.24 \\
Over-Segmentati-on & 3.71 & 7.28 & 17.67 \\
The error rate & 1.22 & 6.91 & 7.02 \\
Overall success rate & 96.81 & 85.81 & 75.22 \\
\hline
\end{tabular}


Table 4. Summary of single factor value

\begin{tabular}{lcccc}
\hline Groups & Count & Sum & Average & Variance \\
\hline Col1 & 4 & 195.4 & 48.85 & 2947.62 \\
Col 2 & 4 & 180.04 & 45.01 & 1922.30 \\
Col 3 & 4 & 157.15 & 39.287 & 1040.64 \\
\hline
\end{tabular}

Table 5. ANOVA single-factor value

\begin{tabular}{lcccccc}
\hline Source of Variation & SS & df & MS & F & P- Value & F crit \\
\hline Between Groups & 185.2454 & 2 & 92.6226 & 0.0470 & 0.9543 & 4.2564 \\
Within Groups & 17731.71 & 9 & 1970.19 & & & \\
Total & 17916.96 & 11 & & & & \\
\hline
\end{tabular}

for managing any over-segmentation problems and can solve special challenges, such as those described in Section 2.

\section{Acknowledgements}

The authors would like to thank Prof Dr. Khairuddin Omar of the National University of Malaysia for his assistance and cooperation. This study was funded by the FRGS/1/2014/ICT07/UKM/01/1 grant entitled "Improving Segmentation of Arabic Handwriting by Determination of Neighborhood Using Voronoi Diagrams."

\section{References}

1. Zeki A. Segmentation of Arabic characters using Voronoi diagrams. University Kebangsaan Malaysia, Malaysia. 2008.

2. Ramdan J, Omar K. Comparative Study of Algorithms for Voronoi Diagram Construction on Segmentation of Arabic Hand Writing. Australian Journal of Basic and Applied Sciences. 2011; 5(11):1653-67.

3. Al Hamad H. Over-segmentation of handwriting Arabic scripts using an efficient heuristic technique. International Conferenceon Wavelet Analysis and Pattern Recognition. 2012; 180-5.

4. Zeki A, Zakaria M, Liong C. The use of Area-Voronoi Diagram in Separating Arabic Text Connected Components. Proceedings of 3th the International Conference on Electrical Engineering and Informatics. 2007.

5. Jyothi J, Manjusha K, Kumar MA, Soman KP. Innovative Feature Sets for Machine Learning based Telugu Character Recognition. Indian Journal of Science and Technology. 2015 Sep; 8(24). Doi: 10.17485/ijst/2015/v8i24/79996.

6. Billauer E. Peak detection using MATLAB [Internet]. 2013. Available from: http://www.billauer.co.il.

7. Pechwitz M, Maddouri SS, Margner V, Ellouze N, Amiri H. IFN/ENIT-database of handwritten Arabic words. Proceedings of CIFED. Citeseer. 2002. p. 127-36.
8. Slimane F, Kanoun S, Alimi AM. Database and Evaluation Protocols for Arabic Printed Text Recognition. Proceedings of 10th International Conference on Document Analysis and Recognition. 2012.

9. Al Hamad H, Hamdi-Cherif A. The Arabic Center for Document Analysis and Recognition ( ACDAR ) - Structure and Perspectives. Recent Advances in Information Science. 2010; (1):85-91.

10. Al-ma S, Elliman D, Higgins C. A Data Base for Arabic Handwritten Text Recognition Research. Proceedings of 8th the International Workshop on Frontiers in Handwriting Recognition. 2002; 1(1):117-21.

11. Ramdan J, Omar K, Mady A. Arabic Handwriting Data Base for Text Recognition. Proceedings of the 4th International Conference on Electrical Engineering and Informatics. 2013; 11(13). p. 580-4.

12. Shukla MK, Banka H, Yadav KP. Structural Features Extraction for Devnagari and Bangla Language Documents. Indian Journal of Science and Technology. 2015 Jul; 8(13). Doi: 10.17485/ijst/2015/v8i13/56453.

13. Bataineh B, Abdullah SNHS, Omar K. A statistical global feature extraction method for optical font recognition. Intelligent Information and Database Systems. Springer. 2011; 257-67.

14. Thakare V. Survey On Image Texture Classification Techniques. International Journal of Advancements in Technology. 2013; 4(1):97-10.4 Available from: http://ijict.org/ index.php/ijoat/article/viewArticle/imagetexture

15. Zitova B. Image registration methods: a survey. Image Vis Comput [Internet]. 2003; 21(11):977-1000. Available from: http://dx.doi.org/10.1016/S0262-8856(03)00137-9\nhttp:// www.sciencedirect.com/science/article/pii/

16. Zeki A. The segmentation problem in arabic character recognition the state of the art. 1stInternational Conference on Information and Communication Technologies. 2005; $11-26$.

17. Zeki A, Zakaria MS, Liong CY. Isolation of dots for arabic ocr using voronoi diagrams. Proceedings of the International Conference on Electrical Engineering and Informatics. 2007. p. 199-202. 